\title{
BMJ Open Decision aids for randomised controlled trials: a qualitative exploration of stakeholders' views
}

\author{
Katie Gillies, Zoë C Skea, Marion K Campbell
}

To cite: Gillies K, Skea ZC, Campbell MK. Decision aids for randomised controlled trials: a qualitative exploration of stakeholders' views. BMJ Open 2014;4:e005734. doi:10.1136/bmjopen-2014005734

- Prepublication history and additional material is available. To view please visit the journal (http://dx.doi.org/ 10.1136/bmjopen-2014005734)

Received 20 May 2014 Revised 17 July 2014 Accepted 28 July 2014

CrossMark

Health Services Research Unit, University of Aberdeen, Aberdeen, UK

Correspondence to Dr Katie Gillies; k.gillies@abdn.ac.uk

\section{ABSTRACT}

Objectives: To explore stakeholders' perceptions of decision aids designed to support the informed consent decision-making process for randomised controlled trials.

Design: Qualitative semistructured interviews. Participants were provided with prototype trial decision aids in advance to stimulate discussion. Interviews were analysed using an established interpretive approach.

Participants: 23 stakeholders: Trial Managers $(n=5)$; Research Nurses $(n=5)$; Ethics Committee Chairs $(n=5)$; patients $(n=4)$ and Clinical Principal Investigators $(n=4)$.

Setting: Embedded within two ongoing randomised controlled trials. All interviews conducted with UKbased participants.

Results: Certain key aspects (eg, values clarification exercises, presentation of probabilities, experiences of others and balance of options) in the prototype decision aids were perceived by all stakeholders as having a significant advantage (over existing patient information leaflets) in terms of supporting well informed appropriate decisions. However, there were some important differences between the stakeholder groups on specific content (eg, language used in the section on positive and negative features of taking part in a trial and the overall length of the trial decision aids). Generally the stakeholders believed trial decision aids have the potential to better engage potential participants in the decision-making process and allow them to make more personally relevant decisions about their participation.

Conclusions: Compared to existing patient information leaflets, stakeholders perceived decision aids for trial participation to have the potential to promote a more 'informed' decision-making process. Further efforts to develop, refine and formally evaluate trial decision aids should be explored.

\section{INTRODUCTION}

There is an ethical requirement to obtain informed consent from potential participants before they are enrolled in a randomised controlled trial (RCT). ${ }^{1}{ }^{2}$ As part of the informed consent process, potential trial

\section{Strengths and limitations of this study}

- This study is the first to explore, and evidence, the potential of a decision aid to support decision-making for participating in a randomised controlled trial from the perspectives of a range of stakeholders, including: patients; Trial Managers; Research Nurses; Clinical Principal Investigator; and Ethics Committee Chairs.

- Compared to existing patient information leaflets, this study has shown that trial decision aids have the potential to better engage potential participants in the decision-making process and allow them to make more personally relevant decisions about their participation.

- All the participants in our study were UK based and a self-selecting sample and therefore may hold different views to those in other countries with different social norms and cultures. However, these participants can offer thoughtful and reflective insights into decision aids for trial participation when reflecting on their own trial experience including reflection on existing patient information leaflets.

participants are provided with written information about the trial often in the form of a participant information leaflet (PIL). ${ }^{3}$ The information included in PILs is largely guided by the Declaration of Helsinki, the International Conference on Harmonisation and Good Clinical Practice (ICH GCP) and, in the UK, by national guidance such as the National Research Ethics Service (NRES). ${ }^{2-4}$ As outlined by this guidance the PIL should include largely fact-based information about: the purpose of the trial; procedures; interventions; possible risks and benefits; sources of finance; conflicts of interest; and the researcher's affiliation. ${ }^{3} 4$

Existing PILs may be suboptimal; research has shown that some trial participants (both those considering participation and those actively enrolled) fail to understand key aspects of trial rationale or process. ${ }^{5}{ }^{6} \mathrm{~A}$ range of studies have tested ways to improve 
information provision in the context of trials. ${ }^{6}$ These have tended to focus on the content and structure of the information and measured outcomes such as understanding, recall and trial recruitment. ${ }^{6}$ While improving understanding of the trial is important, informed decision-making about trial participation is complex and likely to require more than just greater understanding of fact-based information. ${ }^{6}$ Furthermore, it has been argued that PILs are 'institutionally scripted' as a means to obtain ethical approval rather than functioning as a tool to support potential participants' decision-making. ${ }^{7}$ As such, the current conceptualisation of 'informed consent' (largely as understanding of information) and how it is enacted (through signing of a consent form) may be overly narrow and require broadening to consider the importance of deliberation and determination in the decision-making process for trial participation. ${ }^{89}$

Evidence from the treatment and screening decisionmaking literature has highlighted that certain key items are important for making 'good' decisions. ${ }^{10}{ }^{11}$ For example, being able to consider alternative options (in the context of trial participation this may be another intervention or may be usual care), making trade-offs and evaluating potential outcomes of the decision and consideration of what those outcomes mean personally for that individual. These items, and others, are often included in decision aids, which actively encourage people to participate in decisions about treatment that involve weighing up associated benefits and harms often when there is clinical uncertainty. ${ }^{10}$ Decision aids have been developed for a variety of treatment and screening decisions and have been shown to positively influence several aspects of decision-making. ${ }^{10}$ The items identified as being important for good decision-making are largely lacking from existing PILs for trial participation, ${ }^{12}$ further supporting the contention that existing PILs do not function well as decision-making tools. ${ }^{12}$

The very few published studies that have explored the use of decision aids, or components of them, in the context of trial participation decisions have shown some promise ${ }^{13-15}$ For example, compared to existing PILs, decision aids for trial participation have been shown to improve understanding while not increasing anxiety ${ }^{13}$ and resulted in low levels of decisional conflict and high levels of satisfaction. ${ }^{14}$ Although encouraging, these studies have solely focused on trial participants' perceptions and have not explored other stakeholders' opinions. While trial participants perspectives remain key, replacement of or any amendments to existing PILs would require buy-in from an additional range of stakeholders, such as: developers (eg, Trial Managers); deliverers (eg, Research Nurses and Clincal Principal Investigator); and approvers (eg, Ethics Committees). This buy-in is critical to ensuring that trial decision aids are as effective as they can be (ie, act as a decision support tool to facilitate meaningful conversations that encourage informed decision-making), are implementable and used as intended. Although treatment and screening decision aids have been shown to be efficacious, the main barriers to their effectiveness in a real world setting are a lack of implementation and fidelity of use often as a result of a lack of buy-in at inception from stakeholders. ${ }^{16}{ }^{17}$ Furthermore, previous studies on trial decision aids have not explicitly explored perceptions of the 'new' content (ie, features to improve decisionmaking), which define decision aids as different to existing PILs.

The study reported in this manuscript forms part of a larger programme of work that aimed to systematically develop and pilot (through interviews reported here) prototype trial decision aids. The prototype decision aids were developed through an iterative process informed by the MRCs framework on development of complex interventions. ${ }^{18}$ The process began with establishing the current evidence on the effectiveness of decision aids for supporting decisions about RCT participation. ${ }^{19}$ Next a Delphi study was conducted, with a range of stakeholders, to identify key items for inclusion, ${ }^{20}$ followed by an evaluation of existing PILs using a tool (that contains items assessing key features of 'good' decision-making) to identify areas that were lacking, ${ }^{12}$ drafting of protoype decision aids (informed by previous stages), followed by rounds of revision within the study team. We then undertook an in-depth qualitative study to explore stakeholders' views and perspectives on the specific content of the prototype decision aids and their potential to improve the informed consent process for RCTs the qualitative study reported in this paper.

\section{METHODS}

Development of the prototype trial participation decision aids

Prototype decision aids were developed for two on-going RCTs. The first was a trial comparing two surgical procedures for treatment of haemorrhoids (ISRCTN 80061723, date of registration 8 March 2010); and the other a drug trial comparing two active drugs and a placebo for treatment of ureteric stones (ISRCTN 69423238, date of registration 18 November 2010). These RCTs were identified from the portfolio of RCTs managed by the Centre of Healthcare Randomised Trials (CHaRT) at the University of Aberdeen. The content of the prototype decision aids was developed through the iterative process outlined above. The prototype decision aids were enhanced by a graphic designer, at the University of Aberdeen, to improve the visual impact of the tools. The tools were presented as A5 booklets which could be printed or read as a PDF document.

\section{Exploration of stakeholders' perceptions of trial decision aids}

An open-ended topic guide was developed to elicit accounts of participant's view of the prototype decision aids (see online supplementary additional file 1). The topic guide was informed by literature on content items 
for decision aids and explored the key differences between decision aids and existing PILs. ${ }^{11} 12$ Moreover, items identified as contentious in earlier work $^{20}$ were also further explored (eg, use of experiences of others). The guide, and subsequent analysis, were organised around views of existing patient information leaflets; views about the prototype decision aids with specific exploration of their potential to support the decisionmaking process. Semistructured interviews were conducted with different stakeholder groups (including patients, Trial Managers, Research Nurses, Ethics Committee Chairs and Clinical Principal Investigators involved with both trials) to explore perspectives about the use of decision aids in a trials context.

\section{Sampling and recruitment}

Potential participants from the Trial Manager, Research Nurse and Ethics Committee Chair stakeholder groups were identified through email list serves (Trial Managers: UK Clinical Research Collaboration Trial Managers listserv ( $\mathrm{n}=501)$; Research Nurses: Scottish Research Nurse and Coordinators Network listserv ( $\mathrm{n}=198)$; Ethics Committee Chairs: National Research Ethics Service committee chair listserv $(\mathrm{n}=88)$ ). Patients who would be eligible for each RCT were identified and contacted by a Research Nurse working at the lead site for each of the RCTs $(n=20)$. Clinical Principal Investigators for both of the RCTs were sent an email invite and asked to respond to the lead researcher $(\mathrm{KG})$ to express interest $(n=40)$. Prospective participants were sent a letter of invite with a slip to return, or email response, to express interest. Interested participants were sent full information about the study (in the form of a participant information leaflet), and a consent form and were provided with an opportunity to discuss the research project and have any questions answered before making a decision. Ethics Committee Chairs, Clinical Principal Investigators, Research Nurses and Trial Managers who were recruited for interview were sent a copy of both decision aids to review. Recruited patients were only sent the decision aid relevant for their condition. Recruited participants were given the choice of a face-to-face or telephone interview. All participants provided written consent.

\section{Data collection}

One author (KG) conducted the interviews between April 2012 and July 2012. Only one patient participant chose a face-to-face interview, which was conducted at the University of Aberdeen as agreed by the participant and the researcher, all other participants requested telephone interviews. Interviews were audio recorded, transcribed verbatim and anonymised. At the start of the interviews, participants were encouraged to provide their views and perspectives on existing patient information leaflets for clinical trials and discuss their experiences of participating in clinical trials or reviewing clinical trial information, as appropriate. All participants were then asked about their views of the prototype decision aids and how they might, or might not, support a decision about trial participation (see online supplementary additional file 1).

\section{Data management and analysis}

A thematic content analysis of the transcripts was conducted. An established interpretive approach was used whereby following familiarisation with the transcripts, a priori and emergent themes were identified, discussed and agreed by the research team. ${ }^{21}$ As many of the interview questions were developed around predetermined themes of interest (ie, those relating to specific content and purpose of trial decision aids ${ }^{11}$ ) there were not many emergent themes identified. However, the meaning and importance attached to each of the predetermined themes was emergent. Two authors (KG and $\mathrm{ZS}$ ) independently reviewed transcripts and documented the major emerging themes. A thematic framework was subsequently generated, and agreed through discussion with all authors, which detailed codes for labelling textual data related to the major themes and subthemes. Codes with specific relevance to decision aids (and items which define them as being distinct from existing PILs) were used as a priori codes for key parts of the interview transcripts. ${ }^{11}$ Transcripts were subsequently coded by one author $(\mathrm{KG})$, in which the thematic framework was applied systematically to the textual data. This process was managed through the use of text management software (NVivo V.10). This facilitated data organisation which promoted further analytic consideration through constant comparison of data both within and across the stakeholder groups, this was conducted by two authors (KG and ZS) and identified key differences between the groups and identified consensus on the importance of the potential of decision aids across all groups. Relevant quotes representing interviewees' considerations were selected to illustrate the results.

\section{Ethical approval}

The study was approved by the North of Scotland Research Ethics Committee 1 (REC Reference Number 09/S0802/105) and National Health Service (NHS) Grampian Research and Development department (Reference Number 2009HS002). All interview participants provided their signed consent, which included consent for anonymised quotes from their interviews to be published.

\section{RESULTS}

\section{Sample characteristics}

Fifty individuals contacted the researcher (23 Trial Managers; 10 Research Nurses; 8 Ethics Committee Chairs; 5 patients and 4 Clinical Principal Investigators) and 23 were interviewed. Response rates varied across the groups: $5 \%$ for Trial Managers; $7 \%$ for Research Nurses; $9 \%$ for Ethics Committee Chairs; 25\% for patients (1 subsequently declined participation); and 
$10 \%$ for Clinical Principal Investigators. In those stakeholder groups where more participants responded than were required for interview, participants were sampled purposively based on affiliation with registered UKCRC clinical trials units and further stratified for geographic location. The number of participants in each group was decided based on a predetermined judgement that each group should contain a similar number and be informed by the numbers interviewed in the patient group $(n=4)$. The interviews ranged from 40 to $80 \mathrm{~min}$. We deemed this sample size to be sufficient to identify a range of experiences and views that would generate a manageable amount of data for in-depth analysis within the timescale of this project. ${ }^{22}$

A brief description of the participants is provided in table 1 . They included 12 women and 11 men, aged from 35 to 80 , who were from the following stakeholder groups: Trial Managers $(\mathrm{n}=5)$; Research Nurses $(\mathrm{n}=5)$; Ethics Committee Chairs $(\mathrm{n}=5)$; patients $(\mathrm{n}=4)$ and Clinical Principal Investigators $(n=4)$. Twelve of the sample had experience of working for an NHS organisation and seven worked within Universities. Experience of working in clinical trials (which could be as a recruiter, a Trial Manager, a reviewer of ethical applications of trials) ranged from 3 to 20 years. The majority of the group $(n=21)$ had no previous experience of decision aids but all stakeholders had previous exposure to PILs for trials. The themes described below were largely identified a priori so as to provide a predetermined

\begin{tabular}{lr} 
Table 1 Characteristics of interviewees \\
\hline \multicolumn{2}{l}{ N (\%) } \\
\hline Stakeholder group & \\
$\quad$ Trial Manager & $5(22)$ \\
Research Nurse & $5(22)$ \\
Research Ethics Committee (REC) Chair & $5(22)$ \\
Clinical Principal Investigator & $4(17)$ \\
Patient & $4(17)$ \\
Gender & \\
Male & $11(48)$ \\
Female & $12(52)$ \\
Age (years) & $8(35)$ \\
40 and under & $10(43)$ \\
$41-60$ & $5(22)$ \\
61 and above & $7(37)$ \\
Experience of working in clinical trials (years) & \\
< 10 & $12(63)$ \\
$\geq 10$ & $7(37)$ \\
Location (University or NHS)* & $12(63)$ \\
University & \\
NHS & $21(91)$ \\
Previous experience with decision aids & $2(9)$ \\
None & $0(0)$ \\
Limited & \\
Experienced & \\
${ }^{*}$ Patients (n=4) not included in this category. & \\
NHS, National Health Service. & \\
&
\end{tabular}

exploration of the key content items that differ between existing PILs and decision aids for trial participation. Owing to the predefined areas of importance for investigation informing the topic guide, all themes were discussed by all stakeholder groups but the extent to which their opinions converged differed between groups and across themes.

\section{General impressions of the trial decision aids compared to existing information leaflets}

The majority of stakeholders across all groups perceived that, in principle, trial decision aids were beneficial and an improvement on existing PILs. There was a perception that they provided a 'balanced', unbiased picture, that they were uncomplicated and that they could proactively facilitate more engagement in the decision, compared to existing PILs.

it's very well balanced and I think that's really important because it's not leading anybody in any one direction. And I think that's an excellent part of the whole booklet itself. (Patient 3)

...I think that they [decision aids] are very, very straight forward actually, that as I've said before the patient information leaflets are very wordy things and they have a lot of information to impart to patients and sometimes they will switch off after the third paragraph. (Research Nurse 1)

...there's not just an information sheet; there's a decision making tool to help the patient make decisions, rather than it just being a passive thing of read the information leaflet... whereas this is actually making them work through and think about it, and this is obviously the biggest change and I do think this would be of a benefit. (Trial Manager 2)

Although most of the initial perceptions of the decision aids were positive, some participants, from the Research Nurse and Clinical Principal Investigator groups, did feel that the use of a decision aid could potentially overcomplicate the decision process in this context by providing more information and potentially raising concerns.

My concerns were that sometimes people feel that the patient information sheet alone is onerous, so adding something else on might actually put some people off.... just that it might increase fear or uncertainty. It almost makes the decision bigger, by adding in this decision making tool. (Research Nurse 5)

However, these perceptions were from the minority of participants within these stakeholder groups, with most of the group expressing agreement of the improvement of these decision aids compared to existing methods.

\section{Perception of trial decision aid content}

This section of the paper reports the findings relating to specific aspects of the decision aids which are not 
routinely included in patient information leaflets for trial participation.

\section{Provision of information about positive and negative features of taking part in the trial}

The trial decision aids included information on both the advantages and disadvantages of both options (participating in the trial or not) whereas existing PILs generally only cover issues relating to trial participation. ${ }^{12}$ There were varied views (largely across and within the Research Nurse, Trial Manager and Ethics Committee Chair groups) expressed when participants reflected on whether the information included about positive and negative features of participating in the trial or not was balanced. Some recognised this was a new addition to the standard information and felt the section was well balanced and would be helpful for potential participants to make an informed choice about participation.

I think this does just outline the different variables really that, you know, there are disadvantages about taking part in clinical studies and there are disadvantages about not. It's an interesting new thing as far as I can see, I've not see anything quite that descriptive before. (Research Nurse 1)

Other participants felt that while the overall concept of providing information about both options was advantageous, some of the included information about advantages and disadvantages of options could be deemed as being potentially coercive. This was a view held by most of the Trial Managers, Research Nurses and Ethics Committee Chairs.

And I thought that they [sections on advantages and disadvantages participating in the trial or not participating] were quite helpful.... I did think that one of the sentences [You will receive extra personalised care and attention from Research Nurses by taking part in the trial] possibly was a bit over-emotive. (Trial Manager 4)

Even though many participants agreed that advantages and disadvantages about both options should be included, all of the Ethics Committee Chairs reported some of the language as potentially inappropriate and stated that ethics committees would be uncomfortable with some statements. For example:

I think that there's quite a lot of emphasis on saying to people one of the advantages of taking part is that you'll get some extra care and attention... Now, in a sense that's true given that that is built in to the research procedure, but certainly the committee, we're very... we're very sensitive to anything that could be taken as an extra inducement to take part. And I felt that one or both of these was a bit more emphatic about that and if we'd be reviewing these as a committee I think we wouldn't have been very comfortable with that. (Ethics Committee Chair 2)
However, patients reported this section to be well balanced and felt that this section provided information to illustrate that participating in a clinical trial may provide access to services (whether treatment or follow-up) that would not be available outside of the trial. For example:

...it was honest, it was upfront and I was like...yeah, okay, you won't have to do the questionnaires but yeah, you will get additional care. So there was a little bit of a "We provide you with a luxury service" or you just get the MOT when we're ready for it. So, it was quite a good inducement to take part. (Patient 3)

\section{Presentation of probabilities}

Methods used to present probabilities of outcomes associated with interventions across the two prototype trial decision aids were varied according to reported methods of good practice for decision aids ${ }^{11}$ (see figure 1). Participants were asked to compare where appropriate. There was recognition among participants in all stakeholder groups that presenting complex probabilistic information to potential trial participants is challenging and that individuals have varied preferences and understandings of this type of information, especially within the context of clinical trials and the interventions they are testing.

I think it's a good way of presenting it [risks] in a different way. I think presenting risk as words and as numbers and as something visual is going to help. I think in the end it's still a very hard thing for people to understand, as I said, at a personal level. (Ethics Committee Chair 3).

A couple of participants, from the Clinical Principal Investigator and Ethics Committee Chair groups, raised the importance of placing risk within the context of familiar activities as an effective way to allow potential participants to make judgements about the risks they are willing to take.

\begin{abstract}
...you could say, "This list does look long and worrying but actually these side-effects don't occur very often. By comparison if we listed all the side-effects of paracetamol these are the things you would be told about" and you could say very commonly without any problem at all. (Clinical Principal Investigator 2)
\end{abstract}

\section{Methods for clarifying and expressing values}

The majority of stakeholders across all groups felt that values clarification exercises included in the trial decision aids (see figure 2), which allow patients to trade-off positive and negative features of the decision to facilitate personally meaningful decision-making, were helpful and that they had the potential to facilitate the decisionmaking process.

I mentioned that the pros and cons is very, very good, I think that that would help a lot of people make decisions and it talks about what would happen to me if I 


\begin{tabular}{|c|c|c|c|}
\hline & symptom & $\begin{array}{l}\text { number } \\
\text { of people } \\
\text { affected }\end{array}$ & $\begin{array}{l}\% \text { of } \\
\text { people } \\
\text { affected }\end{array}$ \\
\hline COMMON & $\begin{array}{l}\text { - dizziness } \\
\text { - headache } \\
\text { - constipation }\end{array}$ & $\begin{array}{l}\text { less than } \\
1 \text { in } 10 \text { but } \\
\text { more than } \\
1 \text { in } 100\end{array}$ & $1-10 \%$ \\
\hline UNCOMMON & $\begin{array}{l}\text { - rapid heartbeat } \\
\text { - runny itchy nose } \\
\text { - diarrhea } \\
\text { - nausea } \\
\text { - vomiting } \\
\text { - indigestion } \\
\text { - rash } \\
\text { - } \text { abnormal ejaculation } \\
\text { - increased urination } \\
\text { - fainting } \\
\text { - mood changes. }\end{array}$ & $\begin{array}{l}\text { less than } \\
1 \text { in } 100 \text { but } \\
\text { more than } \\
1 \text { in } 1000\end{array}$ & $0.1-1 \%$ \\
\hline RARE & $\begin{array}{l}\text { - pins and needles } \\
\text { - } \quad \text { impollen gums } \\
\text { imce }\end{array}$ & $\begin{array}{l}\text { less than } \\
1 \text { in } 1000 \text { but } \\
\text { more than } \\
1 \text { in } 10000\end{array}$ & $0.01-0.1 \%$ \\
\hline VERY RARE & $\begin{array}{l}\text { - feeling of weakness, } \\
\text { lethargy } \\
\text { - eye pain } \\
\text { - shortness of breath } \\
\text { - prolonged painful } \\
\text { - erection, } \\
\text { - swelling of lips, } \\
\text { face and neck }\end{array}$ & $\begin{array}{l}\text { Less than } 1 \text { in } \\
10000 \text { or rate } \\
\text { unknown }\end{array}$ & $\begin{array}{l}\text { Less than } \\
0.01 \%\end{array}$ \\
\hline
\end{tabular}

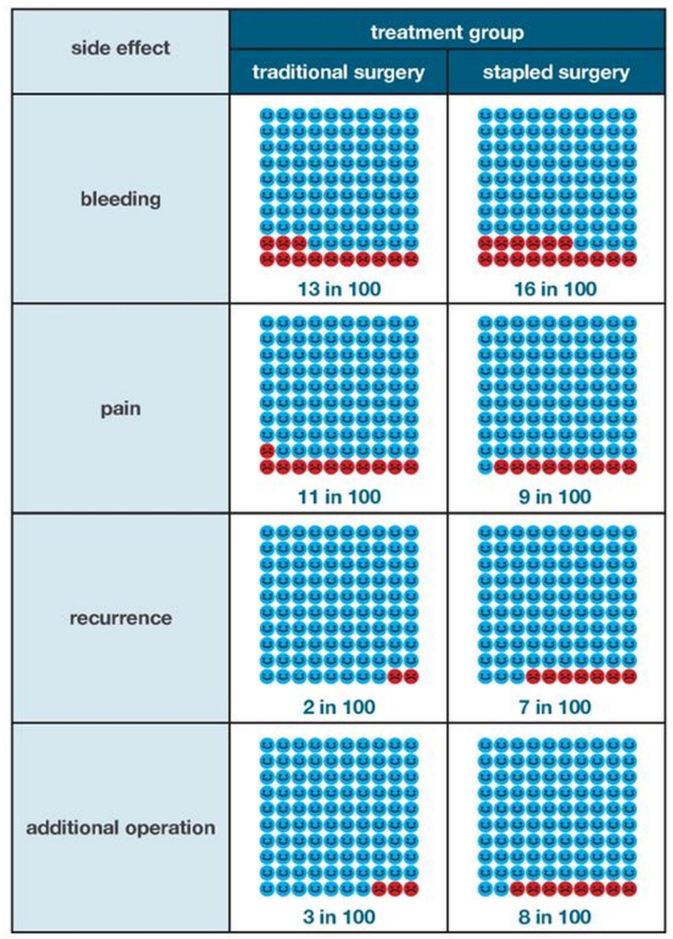

Figure 1 Example of content items from prototype trial decision aids: presenting probabilities section.

didn't take part in this study as well so that's something that we don't, well we say "Oh well that's Ok, you'll just get the standard course of treatment" is there anything negative about me not taking part, that's important to emphasise that as well. (Research Nurse 4)

A significant benefit of values clarification exercises that was highlighted by participants was their potential to allow trial participants to make personally relevant decisions by weighing up what matters most to them, within the context of the clinical trial.

I think it would probably be quite useful just to have that let them weigh that up, whether they want to take part or not. (Clinical Principal Investigator 3)

And that's very powerful, they're making a decision that feels to them very fair because they've done a weighting process around it. So I really, really liked this. (Research Nurse 5)

However, a minority of participants (mainly Trial Managers) felt that the exercises themselves, or aspects of them (such as the term 'worksheets' and the lengthy instructions for completion), would not be helpful and could be perceived negatively.

... I don't know, it just made me think you know patients thinks, "Ah worksheets, am I going to have to fill in loads of stuff?". (Trial Manager 1)

Yet the patient group all perceived these exercises as being helpful and beneficial for their decision-making, acting as a guide to take them through the advantages and disadvantages of trial participation.

I find the little piece at the back, the pros and cons table, or pros and cons balance graphic, quite useful. It did help me come to my conclusion, the pros and cons one, because I answered all the questions and highlighted my answers. I found that really quite interesting. (Patient 2)

However, one of the patients and participants across the other stakeholder groups did highlight that there may be a need for the values clarification exercise to provide a 'score' or objective decision with regard to trial participation.

It's like...there's not a scoring system, so...big benefit, no benefit, so I don't know actually where that would come out. There's no-what's my weighting? (Patient 3).

\section{Structured guidance in deliberation}

Decision aids should provide steps to assist the patient in making a decision, which may include suggesting ways to talk through the decision with health professionals and include tools (worksheets or question lists) that would allow discussion with others. ${ }^{11}$ Participants across all groups stated that the identified steps for making a decision (a list of 6 items outlining the process) that were highlighted in the decision aid (see figure 3) were a helpful addition.

I think putting out how somebody might make a decision. You know, the six points [decision guidance]. And I 
Figure 2 Example of content items from prototype trial decision aids: methods for clarifying and expressing values.

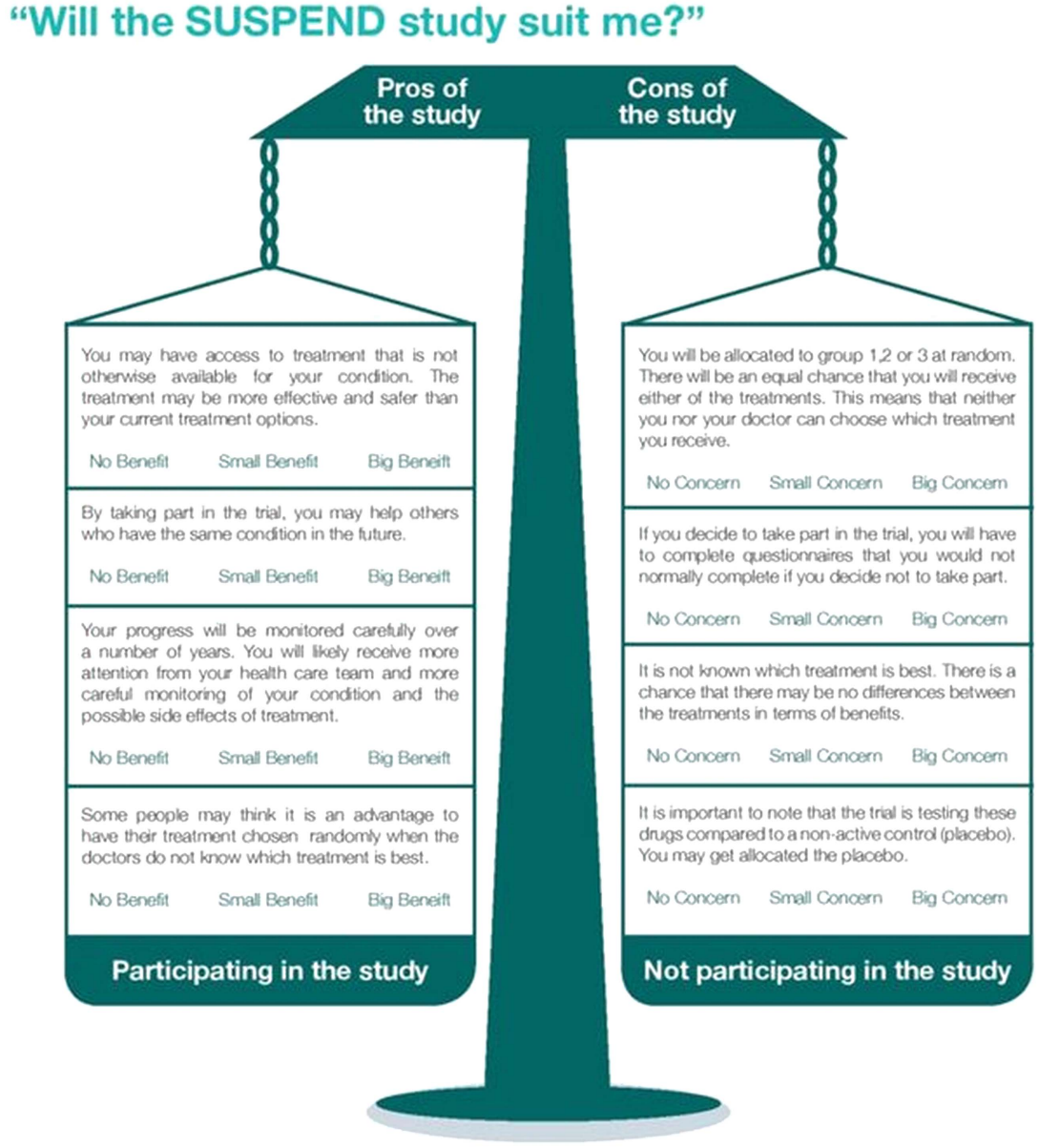

Any further questions?

\section{What are you leaning towards?}

Participating in the study $\star \star \star \star \star \quad$ Not participating in the study think setting all of this... I was pleased that when I read it through. (Ethics Committee Chair 4)

I do think that's good; rather than giving them all the information and then saying "Right, now it's up to you to make a decision." it almost leads them through to actually think: right...it's like making it a much more active decision rather than just reading the leaflet and chucking it away; their actually having to think about the questions in their head. (Trial Manager 1)

There were also positive reactions to the 'notes' page (included as a way to promote question asking and deliberation, which was a blank page titled 'notes'). Participants felt this would facilitate the decision-making process by enabling potential trial participants to ask questions, highlight areas where they need more support to make their decision and reflect on following their decision-making.

And what I thought was excellent, and really this is great, was that you gave room for notes, you know for patients to make notes. It just gives permission for them to be able to do that. And what I thought was, at every time point where you're maybe asking them to go through their decision, put in a blank page which says 'notes', because I just think that is really helpful and it facilitates them actually making notes that they can return back to-"What was my thinking around this?" (Research Nurse 2)

In addition, members of all groups apart from the Clinical Principal Investigators commented on aspects of the decision aid being repetitive. One of the patients stated the following with regard to the structured guidance for decision-making: 


\section{MAKING A DECISION}

The previous pages have outlined the main options available to you. The following steps may help you to make a decision about whether or not to participate in the eTHoS Study.

The decision making process can be helped by following these 6 steps:

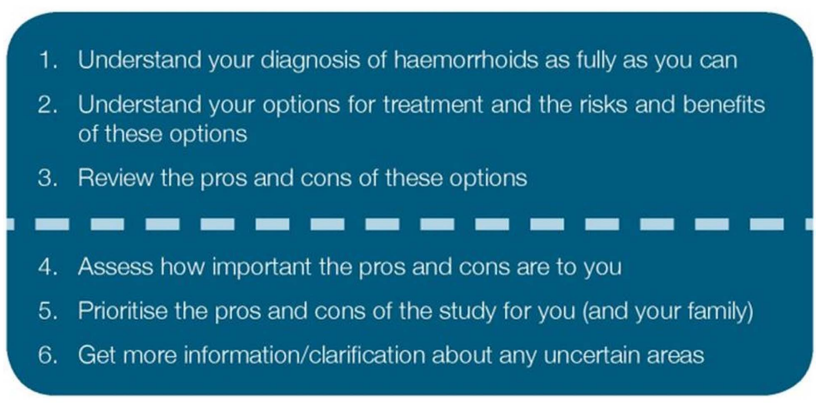

The information in this booklet has already taken you through steps 1-3. To help you complete steps 4-6 and come to a decision that suits you best, we have provided some experiences of others who did or did not take part in the trial and a worksheet for you to think through and complete if you wish (see overleaf).

Figure 3 Example of content items from prototype trial decision aids: structured guidance in deliberation: decision-making steps. (adapted from Juraskova et $\mathrm{al}^{13}$ )

It's very clear. I would, the only comment I would make on that is it's probably repetitive of what's gone on throughout the whole book...But I wouldn't say it would drive me to take that away, not take it out. I just felt that, you know, I'd read most of that and understood most of 1 to 6 in the preceding narrative. (Patient 1)

\section{Experiences of other potential trial participants}

Experiences of others (or patient stories) are sometimes included in treatment decision aids and, if included, should represent a range of experiences, both positive and negative. ${ }^{11}$ Although there were mixed views expressed, most thought the inclusion of other participants' experiences was a helpful addition as the general perception was that people are often interested in what their peers have done and that this could help to normalise trial participation.

It is like a big Expedia or a trip adviser thing, you are always interested in the other people's experiences. Yes actually I think its something that we've not really thought about before, that you are not alone here, that there are hundreds and thousands and millions of people participating in clinical trials all the time so to get a wee bit of feedback from them, yes, yes no I like that. (Research Nurse 1)

Some of the Research Nurse and Clinical Principal Investigator participants reported that trial participants often ask them what other patients have done and that usually there is some dialogue around those experiences.

Yeah, it's [being asked what others think] not infrequent. "What do your other patients think, Mr X?" I usually say, "They often want to get involved." "Oh, well okay then." It's slightly interesting, and a bit bizarre, but there is a bit of team play in that I think. (Clinical Principal Investigator 4)

They say, "What's the uptake of others? Are they all taking part or not?" And I say, "The majority take part in a study; some don't for various reasons. And some of those reasons are personal to that patient: they're too far away, they don't want to come back to the follow-up, they hate hospitals, they don't want to ever come back after this-that type of thing." (Research Nurse 4)

It was also highlighted that experiences of others may enable participants to ask questions by highlighting aspects they may not have previously been considered.

but what it at least does is it encourages them to ask questions because these guys have already identified experiences that they have had. (Research Nurse 1)

Despite generally positive views about the inclusion of others' experiences, there were some queries raised from Trial Managers and Research Nurses, with regard to how the experiences from other trial participants would be generated for inclusion in a trial decision aid given that information leaflets are developed before any participants have entered, or refused, the trial.

So I was a bit unsure how that was all going to work because either you make it generic and it's just about patients who have participated in other trials, or you wouldn't be able to implement this for any trial until after you've already got some patients in. (Trial Manager 4)

There was a concern from one respondent (an Ethics Committee Chair) who perceived there to be no additional value by including experiences of others and that it complicated the process by introducing the perspectives of others when ultimately the decision lies with that individual and should be based on their own values and preferences.

[I'm] Not sure it doesn't... just that it doesn't cloud the water, it was their decision at the end of the day. (Ethics Committee Chair 5)

\section{Amount of information}

There was variation in participants' perceptions about the amount of information and the length of the trial decision aids, with the majority of stakeholders (largely Trial Managers, Ethics Committee Chairs and Principal Investigators agreeing there was too much information and others (patients and Research Nurses)feeling all of the information included was important. There was recognition that the length could be partially attributed to the prespecified regulatory requirements. None of the patients felt there was too much information or that the trial decision aids were too long. For example:

I can't say that I found anything in the book unhelpful. (Patient 3) 
Its difficult because there is so much stuff that is legislated that has to be in, so it is difficult to condense them any less. (Research Nurse 2)

\section{Method of delivery}

The stakeholders in this study had varying preferences, which diverged between and within groups, for how the trial decision aids should be delivered. Some felt that there should be a move towards presenting this type of information online or using other electronic media such as DVDs. However, others felt that providing the information in a booklet format was the best option as this allows people to take it away with them and discuss with others.

You know, if there were a DVD of somebody talking me through this with the diagrams, the presentation, which they could look at in the research room, that would be much better. I'm sure that would be more acceptable to most of them [trial participants]. (Clinical Principal Investigator 4)

I read it quite thoroughly from page to page, and I think that's what it's designed to do, you can take time to read it and make some notes and then consult with somebody else about it, you know? I think the paper document is the best way; the old-fashioned way is the best way, really. (Patient 2)

Some reported that the specific method of delivery is less important and more emphasis should be placed on accessibility.

I think it's important that whatever you use people can access it easily and that if they choose to they can show it to other people outside the place or the room where they made the decision, so they can go over it again. (Ethics Committee Chair 4).

However, participants in the Research Nurse, Trial Manager and Ethics Committee Chair groups identified the importance of context with regard to the trial population being recruited.

Some people were put off by it [computer tablet], but that is just my client group [elderly]. Obviously it is going to really depend on you client group, if it is children, teenagers, people in their twenties, thirties, forties, that's how we live our lives, that is how we expect to receive information nowadays. We certainly don't expect to get it in a paper format. (Research Nurse 2)

\section{The untapped potential of trial participation decision aids} The interviews also focused on participants perceptions of the future potential of decision aids to support decisions about participation. Participants' reflections on this were varied, ranging from improving consent (across all stakeholders) through to increasing recruitment (mentioned by Clinical Principal Investigators and Research Nurses) and retention (highlighted by Research Nurses, Clinical Principal Investigators and Trial Managers) in the trial. However, stakeholders across all groups highlighted a focus on the biggest potential gains to be from improving aspects of the decision-making process such as informedness (which includes an understanding of their involvement and commitment to the trial over time) and opportunities for discussion with others.

To me, it was still open [the decision] right the way through.... But reading this here, right the way through the whole thing you're still feeling, "Well there's still an option, they're still making sure it's ok. (Patient 1).

So I think a tool like this ought and should help people make a better decision, fully informed decision that they can also explain to perhaps their own clinician, certainly to family and friends. (Ethics Committee Chair 3)

I think it's probably making the patient more aware of what's actually involved, and what the commitment will be from the patient. (Trial Manager 1)

There was also recognition, largely by Trial Managers and Research Nurses, that these trial decision aids have the potential to actively engage potential participants in their decision-making process and allow them to make personally relevant decisions that they are able to discuss with others.

...it makes it a bit more personalised, it makes them think about how they would cope with this trial in their life at the time, then I think that would be useful, it would maybe help them think, 'Am I really going to manage this?. (Trial Manager 5)

To empower for decision making, to enfranchise them to make a decision, and to not just get people on study, but to care for people when they're on study, in that this is more helpful to know that they have made a truly well informed decision. And it's something about giving patients the ownership of what they're doing, and I think this is helpful in that. (Research Nurse 5)

\section{DISCUSSION}

\section{Principal findings}

This is one of the first studies to explore perceptions about the potential of decision aids to support decisions about trial participation from the perspective of all key stakeholder groups and provides empirical data on a range of relevant stakeholder perspectives. Furthermore, this is the first study to explicitly investigate stakeholders' views about key content items of decision aids and their appropriateness for decisions about trial participation. Overall, stakeholders felt that the decision aids were an improvement on existing PILs in that they explicitly highlighted that there was a decision to be made about participation in the trial. In addition to this, stakeholders believed that the decision aids also provided ways for potential participants to engage with the decision-making process and make personally appropriate decisions for them as individuals. 
This study explored views about the specific content items that differ between decision aids and existing PILs namely: provision of information about positive and negative features of options; presenting probabilities; methods for clarifying and values; structured guidance in deliberation; and experiences of other potential trial participants. It is important to highlight that while the majority of the stakeholders agreed on specific aspects there were some key differences between the patient group versus the others. For example, patients views differed to the majority of other stakeholders groups with regard to provision of information about positive and negative features of taking part in a trial (specifically with regard to the exacting information contained within the section) in that patients felt it to be balanced but others reported worries about coercive language. In addition, many of the stakeholders felt that the decision aids were too long, but none of the patients reported this with all of them saying that all of the information was important. These findings (which must be considered within the context of this study ie, patients may be different the general population) should serve as a reminder that when developing decision aids for trial participation, while all stakeholder views are important, patients views must be placed at the core.

In principle, the general concept of providing information about positive and negative features of options (ie, to participate or not) was received positively and was felt to provide balance to the decision by highlighting all features. However, some participants expressed views that some of the language was weighted, or may allow participants to attach value to, and could be deemed as potentially coercive. Therefore, it would be important in future decision aids for trial participation to ensure that neutral statements are incorporated. A recent study has illustrated the potential bias that can be introduced into trial participants' decision making when the framing effects of language are not addressed. ${ }^{23}$

The section on presenting probabilities was well received by all stakeholders and was stated to be an improvement on current PILs. However, it served to further highlight that individuals have preferences for the way probabilistic information is presented and that there is no 'one size fits all' approach. This is of particular importance when considering that understanding and perception of risk within clinical trials can be a significant influence on the decision to take part or not. ${ }^{24}$ Although there is a wealth of literature on how best to communicate probabilistic information in a treatment and screening context, this does not exist for decisions about trial participation where often due to the inherent nature of trials, much of this information is not known and the layers of risk are greater (eg, risk of the trial vs risk of treatment, risk of outcomes associated with both interventions, risk of randomisation, etc). Therefore, further research to identify how this can be undertaken effectively, in different trial contexts, are of importance.
The values clarification exercise was reported as a positive addition and provided a way to engage potential participants in their decision making by making them weigh up what matters most to them. One study has measured the extent to which the use of values clarification exercises support (hypothetical) decisions about trial participation and found they lowered ambivalence and decisional uncertainty while improving the clarity of personal values. ${ }^{15}$ Therefore, there is merit in further exploring this type of exercise to support decisions with potential trial participants facing real decisions.

The section on experiences of others was well received by most stakeholders, with several saying that potential participants already ask for this type of information. Participant stories about trial participation are already available through public websites such as healthtalkonline and the NIH clinical trials website. ${ }^{25}{ }^{26}$ However, as yet there is no evidence as to the benefit or harm of including this type of information on people's decisionmaking. While patient stories may be an effective way to increase engagement with the information, there are concerns that people will make decisions based on others values rather than their own. ${ }^{27}$ As such, further research is required to determine whether and how they can be used in this context.

None of the patient group expressed the view that there was too much information incorporated, a finding mirrored by an earlier study exploring patients perceptions of a trial decision aid for radiotherapy for prostate cancer. ${ }^{14}$ However, most of the other stakeholder groups thought the decision aids might be too long. Some stakeholders attributed the amount of information to the guidance requirements for content of informed consent information and recognised this as a barrier against keeping information materials concise. A recent review highlighted the lack of evidence, from a participant's perspective, to support inclusion of many of these prerequisite items in trial information. ${ }^{28}$ However, within the context of a decision aid, stakeholders have agreed that many items required for informed consent (as defined by the regulatory guidance) and items required for informed decision-making (as defined by the International patient decision aid standards) are important and should be included. ${ }^{20}$ Therefore, ways of presenting this information more succinctly need to be explored alongside real-time decision-making by real patients to explore which information is most valued.

The Ottawa Decision Support Framework (ODSF) recommends that during the development and piloting process for decision aids, end users are engaged and their preferences for delivery of the intervention are incorporated. ${ }^{29}$ During this study we elicited participants' views with regard to the most appropriate method of delivery. Stakeholders' perceptions varied in this regard, with some believing that online or electronic methods were best and others believing paper based was optimal but certainly the context and preferences of the end users should be considered. Other studies have 
shown that patients deliberating informed consent for elective surgery had preferences for methods of information provision, with younger patients preferring internet-based information and older patients preferring paper-based information ${ }^{30}$ providing further justification for engaging with users at the outset. However, it should be highlighted that a recent systematic review found equivocal evidence with regard to effectiveness of audiovisual interventions to enhance trial knowledge (during informed consent) but the authors highlight the need to involve consumers in intervention development. ${ }^{31}$ These findings are important for development of decision aids but also for PILs more generally. As such, trial participants and trial staff (eg, Research Nurses, Clinician Principal Investigators) should be engaged during development of trial decision aids to ascertain the best mode of delivery in the trial population. Moreover, if the mode of delivery is novel it may also be worth engaging with ethics committees early in the process.

Overall, these findings complement the previous preliminary work on decision aids for trial participation in that they show that patients perceive these tools as useful and more helpful (compared to existing PILs) in terms of making a well-informed, balanced, personally relevant decision. ${ }^{13}{ }^{14}$ However, our results also contribute additional insights through the involvement of a wide range of stakeholders, which include perspectives from those involved in developing, delivering and reviewing information for patients considering trial participation. Moreover, these findings contribute to the wider literature on participants and stakeholders sense-making of research participation with respect to what it means for them as individuals. For example, a study by Townsend and Cox identifies the importance of the 'meaning' of research participation (including trials) for participants, implicitly underpinned by their individualised context and which transcend the entire participation trajectory, not just the point of consent. ${ }^{32}$

\section{Strengths and weaknesses}

A significant strength of this study was the elicitation of views from a diverse stakeholder group, including: patients; Research Nurses; Trial Managers; Clinical Principal Investigators; and Ethics Committee Chairs. This forms of multistakeholder engagement is promoted as international best practice by the ODSF. Two other studies have explored perceptions of decision aids for trial participation and highlighted their potential benefit, but this previous work has focused only on patients. ${ }^{13} 14$ While patient perceptions are key, as they are the decision-makers, it is important to explore the views of others involved in the informed consent process who would be responsible for developing, endorsing, reviewing and delivering these decision aids. Many of the barriers to implementation of decision aids for treatment decisions relate to 'process' aspects, which may be less relevant for trial decision aids due to a regulatory requirement to provide information in the informed consent process. As such, decision aids for trials would slot in to the existing informed consent process but would require additional training of those delivering to ensure fidelity of use. However, if there is a lack of buy-in and endorsement from those involved in the informed consent process, the decision aids may not be implemented as intended that is, tools to support decision-making that also enable conversations about treatment (and in this context trial participation) to be created and discussed in a meaningful way. Therefore, it is critical to engage with end-users during development.

A further strength of this study was the decision to explore stakeholders' perceptions of key decision aid content items a priori, rather than exploring only general perceptions. This is of particular importance when considering that it is these items which define decision aids as being different to existing PILs.

It may be that the specific trial contexts may have influenced participants' perceptions of the decision aids. However, several sections were written from a generic perspective and were not specific for the individual trial context, which included both a chronic and an acute condition. Moreover, the majority of the stakeholder groups (Research Nurses, Trial Managers and Ethics Committee Chairs) were not directly involved with the trials in which the decision aids were set and the data suggest that their perceptions were being considered commonly across decision aids more widely rather than the exacting information for each trial pilot decision aid presented. All the participants in our study were UK based and therefore may hold different views to those in other countries with different social norms and cultures. However, it was felt that focusing on the UK was appropriate due to the differences in regulatory requirements and structure of PILs across countries, that is, consent forms for American and Canadian studies tend to be longer than UK forms and contain much of the information being found within UK PILs. In addition, there was an assumption that these decision aids were for adults who had capacity to consent for themselves. It would also be important to explore the usefulness of these tools in other contexts with proxy decisionmakers, including parent of children who are consenting on their behalf. Another potential limitation of our study is that the sample were a self-selecting group of individuals and, especially for the patients, may be different from those in the general population. Indeed the size of each of the stakeholder groups was relatively small. However, it is important to highlight that the participants included in this study can offer thoughtful and reflective insights into decision aids for trial participation when reflecting on their own trial experience including reflection on existing PILs.

\section{Implications for researchers}

Decision aids for trial participation should be developed with meaningful stakeholder involvement. All aspects of 
the information should be balanced. Attention to language is critical to ensure it is not deemed coercive or value laden. Developers should be mindful of the target audience, especially when considering presenting probabilistic information and considering method of delivery. If patient stories are included, how these will be generated and included must be considered. Finally, decision aids for trial participation should be developed and used in ways that allow all users to engage effectively with the information and provide support to decision-makers.

\section{Future research}

While the decision aids explored in this study were perceived as being potentially helpful, it should be noted that these types of interventions (or certainly the aids developed in this study) may be more appropriate to support some RCT decisions than others, we are not proposing a 'one size fits all' model. It is likely that decision aids could be more effective for some trial decisions rather than others for example, where interventions being trialled are very different (like medical management vs surgery), which is also the case for treatment decision aids. ${ }^{10}$ It may also be that the decision aid could be broken up into component parts (values clarification exercises, experiences of others, etc) and used as appropriate (defined by individuals preferences for information) in different contexts to facilitate and support the informed decision-making process. However, this requires further evaluation before recommendations can be made.

In addition, given the limitations of the current conceptualisation of informed consent, it is important to think about how decision aids would be evaluated. For example, if tested in an RCT against existing PILs what outcomes should be measured, how do these outcomes compare to others in existing studies of interventions to improve consent, and what do potential participants think should be measured?

Further research regarding how decisions about trial participation are discussed, engaged with, deliberated over, participated in, supported and executed is required to inform the design of interventions to better support the process. In addition, where much of the previous literature has focused on participants' understanding of trial concepts such as randomisation and blinding, exploration of what participants believe taking part means for them as individuals could also help to develop more tailored approaches to informed consent.

\section{CONCLUSIONS}

Compared to existing PILs, decision aids for trial participation have the potential to promote a more 'informed' decision-making process with regard to consent. It is vital that research efforts, inclusive of all stakeholders, continue to understand how to support potential trial participants' decisions about trial participation (whether it be to enroll or not); how to ensure these decisions are in line with individuals values and preferences and to determine optimal methods to support informed decision-making in this context.

Acknowledgements The authors would like to thank all interviewees who gave their time and considerations on the prototype decision aids. The authors would also like to thank Craig Lee, Graphic Designer at the University of Aberdeen, for his contribution in developing the prototype decision aids.

Contributors KG conceived the study idea. KG, ZCS and MKC were involved in designing the study and developing the methods. KG applied for ethics approval and collected the interview data. KG and CZS conducted the initial analysis and development of the thematic framework, with additional input from MKC. KG directed the full analysis. KG wrote the initial manuscript draft. All authors critically revised the manuscript and approved the final version.

Funding This work was supported by personal fellowship award (to KG) from the Chief Scientist Office of the Scottish Governments Health and Social Care Directorates, grant number [PDF/09/01]. The Health Services Research Unit is supported by a core grant from the Chief Scientist Office of the Scottish Government Health and Social Care Directorates.

Competing interests None.

Ethics approval North of Scotland Research Ethics Committee 2.

Provenance and peer review Not commissioned; externally peer reviewed.

Data sharing statement No additional are data available.

Open Access This is an Open Access article distributed in accordance with the Creative Commons Attribution Non Commercial (CC BY-NC 4.0) license, which permits others to distribute, remix, adapt, build upon this work noncommercially, and license their derivative works on different terms, provided the original work is properly cited and the use is non-commercial. See: http:// creativecommons.org/licenses/by-nc/4.0/

\section{REFERENCES}

1. Beauchamp TL, Childress JF. Principles of biomedical ethics. 5th edn. Oxford: Oxford University Press, 2001.

2. World Medical Association (WMA). WMA declaration of Helsinki: ethical principles for medical research involving human subjects. Ferney-Voltaire: WMA, 2008. http://www.wma.net/en/30publications/ 10policies/b3/index.html

3. International Conference on Harmonisation (ICH). ICH Harmonised Tripartite Guideline: Guideline for Good Clinical Practice E6(R1). Geneva: ICH, 1996. [Step 4 version]

4. National Research Ethics Service (NRES). Information sheet and consent forms: guidance for researchers and reviewers. London: National Health Service, National Patient Safety Agency, 2009.

5. Flory J, Emanuel E. Interventions to improve research participants' understanding in informed consent for research: a systematic review. JAMA 2000;6:1593-601.

6. Nishimura A, Carey J, Erwin PJ, et al. Improving understanding in the research informed consent process: a systematic review of 54 interventions tested in randomized control trials. BMC Med Ethics 2013;14:28.

7. Armstrong $\mathrm{N}$, Dixon-Woods $\mathrm{M}$, Thomas $\mathrm{A}$, et al. Do informed consent documents for cancer trials do what they should? A study of manifest and latent functions. Sociol Health IIIn 2012;34:1230-45.

8. Abhyankar P. Decision making about cancer treatment and clinical trial participation. PhD thesis. Leeds: University of Leeds, 2008.

9. Gillies K, Entwistle V. Supporting positive experiences and sustained participation in clinical trials: looking beyond information provision. $J$ Med Ethics 2012;38:751-6.

10. Stacey D, Bennett CL, Barry MJ, et al. Decision aids for people facing health treatment or screening decisions. Cochrane Database Syst Rev 2011;10:CD001431.

11. Elwyn G, O'Connor A, Stacey D, et al. International Patient Decision Aid Standards (IPDAS) Collaboration. Developing a quality criteria framework for patient decision aids: online international Delphi consensus process. BMJ 2006;333:417.

12. Gillies K, Huang W, Skea Z, et al. Patient information leaflets (PILs) for UK randomised controlled trials: a feasibility study exploring whether they contain information to support decision making about trial participation. Trials 2014;15:62. 
13. Juraskova I, Butow P, Lopez A, et al. Improving informed consent: a pilot of a decision aid for women invited to participate in a breast cancer prevention trial (IBIS-II DCIS). Health Expect 2008:11:252-62.

14. Sundaresan $\mathrm{P}$, Turner $\mathrm{S}$, Kneebone $\mathrm{A}$, et al. Evaluating the utility of a patient decision aid for potential participants of a prostate cancer trial (RAVES-ROG 08.03). Radiother Oncol 2011;101:521-4.

15. Abhyankar $P$, Bekker HL, Summers $B A$, et al. Why values elicitation techniques enable people to make informed decisions about cancer trial participation. Health Expect 2011;14:20-32.

16. Wyatt KD, Branda ME, Anderson RT, et al. Peering into the black box: a meta-analysis of how clinicians use decision aids during clinical encounters. Implement Sci 2014;9:26.

17. Elwyn G, Scholl I, Tietbohl C, et al. "Many miles to go ...." a systematic review of the implementation of patient decision support interventions into routine clinical practice. BMC Med Inform Decis Mak 2013;13(Suppl 2):S14.

18. Craig P, Dieppe P, Macintyre S, et al.; Medical Research Council Guidance. Developing and evaluating complex interventions: the new Medical Research Council guidance. BMJ 2008;337:a1655.

19. Gillies K, Skea Z, Politi MC, et al. Decision support interventions for people making decisions about participation in clinical trials (Protocol). Cochrane Database Syst Rev 2012;(3):CD009736.

20. Gillies K, Skea ZC, MacLennan SJ, et al. Determining information for inclusion in a decision-support intervention for clinical trial participation: a modified Delphi approach. Clin Trials 2013;10:967-76.

21. Ritchie J, Spencer L. Qualitative data analysis for applied policy research. In: Bryman A, Burgess R, eds. Analysing qualitative data. London: Routledge, 1993:173-94.
22. Guest G, Bunce A, Johnson L. How many interviews are enough? An experiment with data saturation and variability. Field Notes 2006;18:59-82.

23. Abhyankar P, Summers BA, Velikova G, et al. Framing options as choice or opportunity: does the frame influence decisions? Med Decis Making 2014;34:567-82.

24. Wray RJ, Stryker JE, Winer E, et al. Do cancer patients fully understand clinical trial participation? A pilot study to assess informed consent and patient expectations. J Cancer Educ 2007;22:21-4.

25. http://healthtalkonline.org/peoples-experiences/medical-research/ clinical-trials/topics

26. http://www.nih.gov/health/clinicaltrials/stories/index.htm

27. Winterbottom A, Bekker HL, Conner M, et al. Does narrative information bias individual's decision making? A systematic review. Soc Sci Med 2008;67:2079-88.

28. Kirkby HM, Calvert M, Draper $\mathrm{H}$, et al. What potential research participants want to know about research: a systematic review. BMJ Open 2012;2:e000509.

29. O'Connor AM, Tugwell P, Wells GA, et al. A decision aid for women considering hormone therapy after menopause: decision support ramework and evaluation. Patient Educ Couns 1998;33:267-79.

30. Bird JH, Biggs TC, Bennett WO, et al. Patient preferences for the method of delivery of preoperative patient information. Bull $R$ Coll Surg England 2013;95:228-30.

31. Synnot A, Ryan R, Prictor M, et al. Audio-visual presentation of information for informed consent for participation in clinical trials. Cochrane Database Syst Rev 2014;(5):CD003717.

32. Townsend A, Cox SM. Volunteering for research: accessing health services through the back door? BMC Medical Ethics 2013;14:40. 